\title{
The effects of capsaicin on the neurogenic hyperreflexic detrusor. A double blind placebo controlled study in patients with spinal cord disease. Preliminary results
}

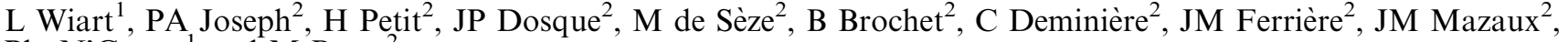 \\ Ph. N'Guyen ${ }^{1}$ and $M$ Barat ${ }^{2}$ \\ ${ }^{1}$ Neuro rehabilitation Unit Center, Tour de Gassies, 33523 Bruges Cedex, France; ${ }^{2}$ Pellegrin University Hospital, \\ 33071 Bordeaux, Cedex France
}

\begin{abstract}
Purpose: Several recent open studies have provided encouraging results as to the efficacy of intravesical installations of capsaicin for neurogenic hyperreflectivity. The present trial was performed to verify these results under controlled conditions. Intravesical installation of capsaicin represents a new therapeutic hope for the treatment of the neurogenic hyperrelfexic bladder.

Method: This randomized, double-blind study compared the results of the intravesical installation of $30 \mathrm{mg}$ capsaicin in $100 \mathrm{ml}$ of $30 \%$ alcohol (experimental group) with those of

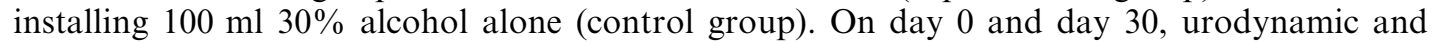
biopsic examinations were performed in all subjects of each group.

Patients: All the subjects included in the study had a functionally disabling form of neurogenic hyperreflexic bladder resistant to the usual therapies. Cystoscopy and retrograde cystography were performed to exclude any patient who presented with a tumor-like lesion or had vesicoureteral reflux.

Results: Twelve paraplegic of tetraplegic subjects, seven women and five men whose average age was 46, were included. Eight had multiple sclerosis, and four had sustained a traumatic spinal cord injury. The patients were randomly separated into two groups of six. Initially, there was no clinical or urodynamic differences in these groups. Installation immediately triggered side effects and during the first 7 days (suprapubic burning sensation, sensory urgency, hot flushes, autonomic hyperreflexia, hematuria) in five of the six subjects in both groups. Bladder biopsy revealed no significant deterioration. On day 30, there was improvement in all of the experimental-group of patients with significant regression of leakage $(P=0.002)$ and of sensory urgency $(P=0.01)$. Only one control subject had amelioration. Urodynamic examination showed a rise in bladder capacity from 172.5 to $312.3 \mathrm{ml}$ in the experimental group, significantly greater $(P=0.03)$ than the rise from 129 to $175.3 \mathrm{ml}$ observed in the control group.

Conclusion: This trial fully confirms the efficacy of intravesical installations of capsaicin, an efficacy obtained at the cost of nonnegligible side effects. An intermediate-term follow-up of this treatment will be necessary before considering more widespread use of this agent.
\end{abstract}

Keywords: capsaïcin; detrusor hyperactivity; neuropathic bladder; multiple sclerosis; paraplegia

\section{Introduction}

Studies on spinal cord injured animals ${ }^{1,2}$ and preliminary studies on a small population of spinal cord injured patients $^{3}$ suggest that capsaicin-sensitive, C-fiber bladder afferents are involved in pathological conditions associated with a neurogenic hyperreflexic bladder. Open trials indicate substantial therapeutic potential for intravesical capsaicin treatment of a neurogenic hyperreflexic detrusor, ${ }^{4-9}$ but controlled trials have not

Correspondence: Dr L Wiart, Tour de Gassies Center, 33523 Bruges Cedex, France yet been published. ${ }^{7}$ We report the results of a doubleblind, placebo-controlled study performed to delineate clinical and cystometric effects of intravesical capsaicin treatment, and to provide further data concerning its potential short-term side effects.

\section{Methods}

Patients

We included in this protocol patients showing severe neuropathic incontinence resistant to currently avail- 
able medical treatments. All of these patients had spinal cord disease, considerable detrusor hyperreflexia. Patients showing progression of neurological symptoms during the previous 6 months were excluded from our study. Those with urinary tract infection and lithiasis were ruled out or were treated before the capsaicin installation. If there was malignancy or ureteral reflux, after cystographic and cystoscopic examinations, patients were excluded.

Twelve patients (seven females, five males) meeting these criteria were included (see Table 1 ). They ranged in age from 38 to 66 years (mean $=45.9$ years). All patients had spastic paraplegia or tetraplegia. Eight had multiple sclerosis and the other four had either a traumatic spinal cord injury. Among the latter, one had T4 complete paraplegia (ASIA Impairment scale (AIS) grade A), one T10 complete paraplegia (AIS grade $\mathrm{A}$ ), one $\mathrm{C} 7$ complete tetraplegia (AIS grade $\mathrm{A}$ ), and one $\mathrm{C} 7$ incomplete tetraplegia (AIS grade $\mathrm{C}$ ).

\section{Procedures}

The ethics committee of Bordeaux University Hospital approved the protocol. All patients gave personal informed consent to participate in the trial and were randomly assigned to either the trial or control (placebo) groups. Synthetic capsaicin was prepared by the Pharmacy School of the University of Bordeaux.

Twenty minutes after an intravesical installation of $40 \mathrm{ml}$ lidocain $(2 \%)$ solution, the patients randomly received installation of capsaicin $(100 \mathrm{ml}$ of $1 \mathrm{mmol} / 1$ [0.3 g/l] solution in $30 \%$ alcohol) or placebo (solution of $30 \%$ alcohol) during $30 \mathrm{~min}$. All installations were carried out at the hospital as outpatients, and they returned home $1 \mathrm{~h}$ after installation.

The subjects were questioned about the clinical effects, especially with regard to incontinence and side effects. Along with these clinical aspects, cystometric parameters volume of the first urge to void, bladder capacity (leak point pressure), and pressure of the detrusor at maximum capacity, were determined at Day 0 (D0) before installation, and 30 days after the installation (D30). Gas cystometric studies $(50 \mathrm{ml} / \mathrm{mn})$ were performed with a Urodyn 2000 apparatus (Dantec Inc.). All of patients underwent a rigid cystoscopic examination 3 weeks before the installation, and at D30. A biopsy specimen was obtained from the dome and trigone of the bladder.

The Mann-Whitney nonparametric test was used to compare the two groups at D0 and D30.

\section{Results}

Initially the two groups presented no significant difference in their general characteristics (Table 1), mode of voiding (Table 2 ), or urodynamic parameters (Table 3).

The installation induced frequent, but well tolerated side effects (no patient came out of the study) with no significant difference between the two groups (Table 4). Among the side effects occurring during or immediately after the installation were a suprapubic burning sensation (in spite of local anesthesia with lidocain), sensory urgency and/or a hot flush in five of six cases in the capsaicin group, and in four of the six control subjects. Only one subject (capsaicin group) developed moderate hyperreflexia, rapidly regressing after treatment with calcium channel blockers. These immediate symptoms are to be distinguished from delayed side effects arising from $12 \mathrm{~h}$ to 6 days after installation: suprapubic pain sensation (SPP) or increasing incontinence (II) or macroscopic haematuria $(\mathrm{MH})$ in five of the six patients in the capsaicin group and in all of the controls. One patient who had

Table 1 General characteristics of patients

\begin{tabular}{|c|c|c|c|c|c|c|}
\hline & Patients & $\begin{array}{c}\text { Sex, } \\
\text { age at } \\
\text { inclusion }\end{array}$ & $\begin{array}{c}\text { Primary } \\
\text { disease }\end{array}$ & $\begin{array}{c}\text { Duration } \\
\text { of } \\
\text { illness } \\
\text { (years) }\end{array}$ & Neurological examination & $\begin{array}{c}\text { Mode } \\
\text { of } \\
\text { voiding }\end{array}$ \\
\hline Experimental Group & $1-$ & M48 & MS & 15 & incomplete tetraplegia & $\mathrm{IC}+\mathrm{VV}$ \\
\hline \multirow{5}{*}{ Capsaicin } & $2-$ & M38 & SCI & 5 & complete paraplegia & $\mathrm{IC}+\mathrm{RV}$ \\
\hline & $3-$ & F55 & MS & 17 & incomplete paraplegia & RV \\
\hline & $4-$ & F46 & MS & 25 & incomplete paraplegia & VV \\
\hline & $5-$ & F45 & MS & 27 & incomplete paraplegia & VV \\
\hline & $6-$ & M66 & SCI & 5 & complete paraplegia & $\mathrm{IC}+\mathrm{RV}$ \\
\hline mean & & 49.6 & & 15 & & \\
\hline Control group & $7-$ & F42 & MS & 24 & incomplete paraplegia & $\mathrm{IC}+\mathrm{VV}$ \\
\hline \multirow[t]{5}{*}{ Placebo } & $8-$ & M42 & SCI & 17 & complete tetraplegia & RV \\
\hline & $9-$ & F40 & MS & 12 & incomplete paraplegia & VV \\
\hline & $10-$ & F37 & SCI & 7 & incomplete tetraplegia & $\mathrm{IC}+\mathrm{RV}$ \\
\hline & $11-$ & M46 & MS & 9 & incomplete paraplegia & $\mathrm{IC}+\mathrm{VV}$ \\
\hline & $12-$ & F46 & MS & 13 & incomplete paraplegia & VV \\
\hline mean & & 42.1 & & 13.6 & & \\
\hline
\end{tabular}

MS: multiple sclerosis, SCI: spinal cord injury, RV: reflex voiding, VV: voluntary voiding, IC: intermittent catheterization 
Table 2 Clinical results

\begin{tabular}{|c|c|c|c|c|c|c|}
\hline \multirow{2}{*}{ Patients } & & \multicolumn{2}{|c|}{ Voiding $(N: 24 H)$} & \multicolumn{2}{|c|}{ Leakages $(N / 24 H)$} & \multirow{2}{*}{$\begin{array}{c}\text { Patient } \\
\text { satisfaction on } \\
\text { D30 }\end{array}$} \\
\hline & & Do & $D 30$ & $D 0$ & $D 30$ & \\
\hline \multirow[t]{2}{*}{ Experimental group } & 1.2 & 7 & 6 & 4 & 2 & Yes \\
\hline & 2.3 & 6 & 5 & 3 & 0 & Yes \\
\hline \multirow[t]{4}{*}{ Capsaicin } & 3.6 & 5 & 5 & 3 & 0 & Yes \\
\hline & 4.8 & 12 & 5 & 5 & 0 & Yes \\
\hline & 5.10 & 8 & 5 & 5 & 0 & Yes \\
\hline & 6.12 & 6 & 5 & 2 & 0 & Yes \\
\hline $\operatorname{Mean}(\mathrm{SD})$ & & $7.3(2.5)$ & $5.1(0.4)$ & $3.6(1.2)$ & $0.3(0.8)$ & \\
\hline \multirow[t]{2}{*}{ Control group } & 7.1 & 16 & 7 & 8 & 4 & Yes \\
\hline & 8.4 & 12 & 12 & 6 & 6 & No \\
\hline \multirow[t]{4}{*}{ Placebo } & 9.5 & 12 & 12 & 3 & 3 & No \\
\hline & 10.7 & 6 & 6 & 6 & 6 & No \\
\hline & 11.9 & 5 & 5 & 5 & 5 & No \\
\hline & 12.11 & 14 & 14 & 6 & 3 & No \\
\hline $\operatorname{Mean}(\mathrm{SD})$ & & $10.8(4.4)$ & $9.3(3.7)$ & 5.1(1.9) & $4.5(1.3)$ & \\
\hline Significance & & $P=0.22$ & $P=0.016$ & $P=0.13$ & $P=0.002$ & \\
\hline
\end{tabular}

Voiding: self catheterization or voluntary micturition; Leakages: non-voluntary micturition

Table 3 Urodynamic Results

\begin{tabular}{|c|c|c|c|c|c|c|}
\hline \multirow[b]{2}{*}{ Patients } & \multicolumn{2}{|c|}{ First urge of void } & \multicolumn{2}{|c|}{ Bladder capacity } & \multicolumn{2}{|c|}{$\begin{array}{l}\text { Pression of detrusor } \\
\text { at maximum capacity }\end{array}$} \\
\hline & DO & $D 30$ & DO & D30 & DO & D30 \\
\hline 1.2 & 80 & 102 & 188 & 243 & 90 & 50 \\
\hline 2.3 & 121 & 107 & 300 & 319 & 50 & 20 \\
\hline 3.6 & 96 & 180 & 109 & 420 & 73 & 47 \\
\hline 4.8 & 51 & 140 & 206 & 340 & 40 & 42 \\
\hline 5.10 & 92 & 159 & 103 & 389 & 70 & 51 \\
\hline \multirow[t]{2}{*}{6.12} & 84 & 141 & 129 & 163 & 82 & 80 \\
\hline & $87.3(22.8)$ & $138.1(29.9)$ & $172.5(75.3)$ & $312.3(95.2)$ & $67.5(19)$ & $48.3(19.2)$ \\
\hline 7.1 & 40 & 105 & 70 & 200 & 27 & 36 \\
\hline 8.4 & 60 & 103 & 75 & 211 & 72 & 80 \\
\hline 9.12 & 68 & 63 & 146 & 165 & 55 & 42 \\
\hline 10.7 & 95 & 85 & 116 & 102 & 61 & 65 \\
\hline 11.9 & 200 & 201 & 206 & 277 & 59 & 65 \\
\hline \multirow[t]{3}{*}{12.14} & 106 & 88 & 161 & 97 & 82 & 80 \\
\hline & $94.8(56.7)$ & $107.5(48.2)$ & $129(52.5)$ & $175.3(69)$ & $59.3(18.6)$ & 61.3(18.6) \\
\hline & $P=0.87(\mathrm{NS})$ & $P=0.10(\mathrm{NS})$ & $P=0.37(\mathrm{NS})$ & $P=0.037(\mathrm{~S})$ & $P=0.4(\mathrm{NS})$ & $P=0.4(\mathrm{NS})$ \\
\hline
\end{tabular}

autonomic hyperreflexia was noted in the control group, and another who had symptomatic lower urinary tract infection in the capsaicin group. Urinary retention was not observed in patients who voided voluntarily.

The cystoscopies and biopsies performed before and after installation showed only non specific aspecific alterations of the vesical mucosa, with no difference between the two groups (the detailed histopathology results will be published separately).

On D30, among the clinical results (Table 2) there appeared to be a significant improvement in the frequency of voiding in the capsaicin group in which the average number of daily catheterizations was reduced from 7.3 to 5.1, compared to a smaller reduction in the control group, from 10.8 to 9.3 $(P=0.016)$. Similarly, the number of daily incidents of leakage fell from 3.6 to 0.3 in the capsaicin group compared to the decrease from 5.1 to 4.5 in the controls $(P=0.002)$. The mode of voiding remained unchanged in all of the patients. These results concur well with the subjective judgment of the patients who were all satisfied in the capsaicin group, compared to only one satisfied patient amongst the controls.

Urodynamically (Table 3), the first urge to void was noted at an average volume of $87.3 \mathrm{ml}$ before and $138.1 \mathrm{ml}$ after the installation of capsaicin compared to 94.8 and $107 \mathrm{ml}$, respectively, in the control group 
(NS: $P=0.1$ ). Similarly, the detrusor pressure at maximum bladder capacity was not significantly lowered in the experimental group (from 67.5 to $48.3 \mathrm{~cm}$ water) compared to the control group (from 59.3 to 61.3$)(P=0.4)$. Bladder capacity significantly improved in the capsaicin group, rising from $172.5 \mathrm{ml}$ on the average on D0 to $312.3 \mathrm{ml}$ on D30, whereas it rose from $129 \mathrm{ml}$ to $175.3 \mathrm{ml}$ in the controls $(P=0.037)$. In this limited study, there did not appear to be any differences between the patients with traumatic spinal injury in comparison with those with multiple sclerosis.

\section{Discussion}

This is the fourth report concerning the use of intravesical installation of capsaicin for the neurogenic hyperreflexic bladder, and the first conducted according to the recommendations of $\mathrm{Nitti}^{7}{ }^{7}$ i.e. double blinded versus placebo. Indeed, although previous works ${ }^{4-7}$ have produced very encouraging results, none permitted, from a strictly methodological point of view, affirmation of the efficacy of capsaicin.

In view of the complexity of the protocol, our trial included only 12 patients, but the two groups were quite homogeneous, initially presenting no differences in general, clinical and urodynamical characteristics. The other four series $4,6,8,9$ included 41 , seven, 12 and three patients, respectively. The installation protocol used in our study was strictly that described by Fowler. 5

We obtained detailed data on side effects (Table 4) which were present in almost every patient at the time of the installation (suprapubic burning sensation, sensory urgency, hot flush and, more rarely, autonomic hyperreflexia) as well as within the following 6 days (suprapubic burning sensation, exacerbation of incontinence, macroscopic hematuria). These effects were also reported by the previous authors, who considered them to be clinically tolerable, as did we. As previously stated, none of our subjects at any point requested to drop out of the study.

One of the principal findings of our study was, as had not been anticipated, the absence of more severe or more frequent secondary effects clinically or histologically documented in the capsaicin group in comparison with the control group. This suggests that the solvent of the installed solution, $30 \%$ alcohol would be just as deleterious to the bladder mucosa as is capsaicin. Further work is called for in order to improve the quality of the solvent. Igawa, ${ }^{10}$ recently observed the absence of autonomic hyperreflexia in two patients having undergone vesical installation with $10 \%$ ethanol alone, whereas it occurred when capsaicin was installed using that solvent. Unfortunately, he made no mention of the other side effects. It is also noteworthy, from a purely methodological standpoint, that the presence of identical side effects in our two groups preserved the double-blind nature of the study consequently reinforcing the reliability of the results.

In terms of clinical efficacy, we found a very marked improvement in leakage and voiding frequency associated with the general satisfaction of the subjects treated with capsaicin. The mode of voiding did not change, but the feeling of greater security in these patients allowed most of them to return to social activities that had previously been limited by their incontinence: shopping, restaurants, movies, the beach, etc. In contrast, in the control group (except for patient 1) the clinical condition remained unchanged without any lasting aggravation. We noticed slight improvement in four of six patients in the control group which could be due to the placebo effect itself or

Table 4 Adverse effect

\begin{tabular}{lcccc}
\hline & Patients & D0 & D1 to D7 & After D7 \\
\hline Experimental Group & 1.2 & SPP,Fl & SPP,II, & UTI \\
Capsaicin & 2.3 & AD,Fl & MH & none \\
& 3.5 & none & SPP,II,UTI & none \\
& 4.8 & SPP,II & SPP,II & none \\
& 5.10 & SPP,2II & SPP,II,MH & none
\end{tabular}

Summary

Control Group

Placebo
SPP:3,II:2,Fl:3,none:1

SPP,F1

none

II

SPP

none

II
SPP:4,II:4,MH:2,UTI:1

UTI:1,none:5

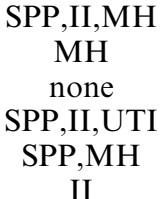

II

SPP: suprapubic pain, Fl: flush, II: incontinence increasing, MH: macroscopic haematuria, UTI: urinary tract infection, AD: autonomic dyreflexia 
to a positive anticipatory attitude of the examiner. This is why a double blind placebo study was necessary. These good results are perfectly compatible with the findings of the open studies: nine out of 12 patients satisfied in Fowler's study, ${ }^{5}$ six of seven for Geirsson, ${ }^{6} 34$ of 41 for de Ridder. ${ }^{9}$ In our study, there were no differences in efficacy between the spinal cord traumatic injury subjects and those with multiple sclerosis, which corresponds to Geirsson's results in seven spinal injury patients.

The urodynamic data confirmed the clinical impression and provided interesting complementary information. The principal improvement concerned the peak bladder capacity which increased by $140 \mathrm{ml}$ on the average in the capsaicin group compared to only $46 \mathrm{ml}$ in the controls $(r=0.037)$. This latter increase could be attributed to a placebo effect, to a possible action of the solvent itself, or to a positive anticipatory effect of the examining physician, but which of these is or are responsible remains uncertain.

These values lie in the range of the results reported in the open studies which found average gains of $50 \mathrm{ml}$ for de Ridder, ${ }^{9} 104 \mathrm{ml}$ for Geirsson, ${ }^{6}$ and $201 \mathrm{ml}$ for Fowler. ${ }^{5}$ An improvement of $140 \mathrm{ml}$ in the bladder capacity should, for a mean hourly diuresis of 50 to $100 \mathrm{ml}$, result in an increase in the intervoiding period of 1.5 to $3 \mathrm{~h}$. This corresponds quite closely with the reduction in voiding frequency found in our trial.

The bladder capacity corresponding to the initial urge to void tended to be improved in our capsaicin group, but the difference with controls was not statistically significant. This may have been due to methodological problems - too few subjects, impossibility of analyzing the urge to void in patients with a complete spinal cord injury. Another possibility that might explain this lack of statistical improvement in initial urge could be the destruction by capsaicin of the nonmyelinated fibers carrying this signal toward the centers of perception. Upscaling of our study with a greater number of subjects might provide answers to this question which was not directly addressed in the previous reports.

The maximal filling bladder pressure, which reflects the vesical contractility was decreased in average of $19 \mathrm{~cm}$ water, but it did not reach significance. Significance could probably be reached with a greater number of patients. This decrease could explain the decrease of sensation of emergency voiding as well as the leakage. Our data are again comparable to previous studies: Geirsson: ${ }^{6} 7 \mathrm{~cm}$ water; Fowler: ${ }^{5}$ $12 \mathrm{~cm}$; de Ridder: ${ }^{9} 15 \mathrm{~cm}$. This was probably due to the persistence of a certain number of nonmyelinated fibers which resisted the installation as was suggested by Chandimarami. ${ }^{8}$ Another hypothesis to explain the maintenace of bladder activity could be the existence of other neurourological pathways responding to other stimulations.
In summary, urodynamically the situation corresponded to one with slightly attenuated bladder contraction and, above all, more delayed volume evoked contraction. This would explain the absence of any urinary retention and the conservation of the mode of voiding.

\section{Conclusions}

This initial double-blind, placebo-controlled trial confirmed the efficacy of capsaicin for neurogenic hyperreflexic bladder, finding clinical and urodynamic results similar to those of the preliminary open studies with tolerable side effects. However, numerous questions remain to be addressed, in particular concerning the long-term maintenance of effects and tolerance.

We are currently extending our double-blind study, progressively increasing the number of subjects. In parallel, we are carrying out an open medium-term follow-up of patients periodically receiving installations.

\section{Acknowledgements}

This study was funded in part by a grant from the Ligue Française Contre la Sclérose en Plaques. We wish to thank CJ Fowler for her help in designing this study.

\section{References}

1 De Groat WC. Mechanisms underlying the recovery of lower urinary tract function following spinal cord injury. Paraplegia 1995; 33: $493-505$.

2 Craft RM, Cohen SM, Porreca F. Long-lasting desensitization of bladder afferents following intravesical resiniferatoxin and capsaicin in the rat. Pain 1995; 61: $317-323$.

3 Dasgupta $\mathrm{P}$ et al. Intravesical capsaicin: its effects on nerve densities in the human urinary bladder. Neurourol Urodyn 1996; 15: $373-374$

4 Fowler CJ et al. Intravesical capsaicin for neurogenic bladder dysfunction. Lancet 1992; 339; 1239.

5 Fowler CJ et al. Intravesical capsaicin for treatment of detrusor hyperreflexia. J Neurol Neurosurg Psychiatry 1994; 57: 169-173.

6 Geirsson G, Fall M, Sullivan L. Effect of intravesical capsaicin treatment on posttraumatic spinal detrusor hyperreflexia and the bladder cooling reflex. Neurourol Urodyn 1994; 13: 346-347.

7 Nitti VW. Intravesical capsaicin for treatment of neurogenic bladder. Lancet 1994; 343: 1448.

8 Chandiramani VA, Peterson T, Beck RO, Fowler CJ. Lessons learnt from 44 intravesical installations of capsaicin. Neurology and Urodynamics 1994; 13: 348 - 349.

9 De Ridder D, Van Poppel H, Baert L. Intravesical Capsaïcin as treatment for detrusor hyperreflexia in multiple sclerosis: results in 41 patients. Neurourology and Urodynamics 1996; 15: $373-$ 374.

10 Igawa Y, Komiyama I, Nishizawa S, Ogawa A. Intravesical Capsaïcin inhibits autonomic dysreflexia in patients with spinal cord injury. Neurourology and Urodynamics 1996; 15: 374-375. 